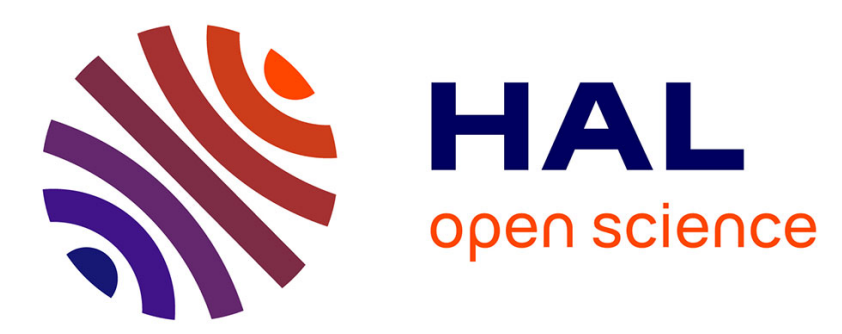

\title{
Challenges in the Computational Implementation of Montagovian Lexical Semantics
}

\author{
Bruno Mery
}

\section{To cite this version:}

Bruno Mery. Challenges in the Computational Implementation of Montagovian Lexical Semantics. New Frontiers in Artificial Intelligence: JSAI-isAI 2016 Workshops Revised Selected Papers, 14, Springer, pp.90-107, 2017, 978-3-319-61572-1. 10.1007/978-3-319-61572-1_7 . hal-01559219

\section{HAL Id: hal-01559219 https://hal.inria.fr/hal-01559219}

Submitted on $10 \mathrm{Jul} 2017$

HAL is a multi-disciplinary open access archive for the deposit and dissemination of scientific research documents, whether they are published or not. The documents may come from teaching and research institutions in France or abroad, or from public or private research centers.
L'archive ouverte pluridisciplinaire HAL, est destinée au dépôt et à la diffusion de documents scientifiques de niveau recherche, publiés ou non, émanant des établissements d'enseignement et de recherche français ou étrangers, des laboratoires publics ou privés. 


\title{
Challenges in the Computational Implementation of Montagovian Lexical Semantics
}

\author{
Bruno Mery^, bruno.mery@u-bordeaux.fr \\ LaBRI \& Université de Bordeaux, France
}

\begin{abstract}
We present and discuss a general-purpose implementation of the process of lexical semantics analysis theorised in the Montagovian Generative Lexicon $\Lambda T Y_{n}$ (hereafter MGL). The prototype software itself constitutes a proof of concept of the MGL theory. The implementation process, as well as the data structures and algorithms, also provide valuable results as to the expressive power required by MGL. While the implementation of terms and types for the purpose of meaning assembly assumed by MGL is in itself straightforward, some lexical phenomena require additional mechanisms in order to process the logical representation in order to take into account implicit common-sense world knowledge. We therefore also present a minimal architecture for knowledge representation, and how it can be applied to different phenomena. The implementation illustrates the validity of the theory, but MGL requires a stronger corpus of types and terms in order to be thoroughly tested.
\end{abstract}

Keywords: Lexical Semantics, Montagovian Generative Lexicon, Knowledge Representation for Natural Language Semantics, Typed Lambda Calculus, Prototype Software.

Pre-print version. All references should be to Springer-published final version, see http://dx. doi org/10.1007/978-3-319-61572-1_7.

\section{Theories and Implementations of Lexical Semantics}

Formal lexical semantics theories aim to integrate to the toolbox of compositional analysis of natural language developed since Montague considerations of (logical) polysemy. Based on original studies such as $[5,10,27]$, then on the Generative Lexicon theory thoroughly developed in [29], there have been many formulations that build upon powerful type-theoretic foundations, with a generative, dynamic account of the lexicon at their heart. Such recent type-theoretic accounts of lexical meaning include Type Composition Logic (TCL) presented in [1], Dynamic Type Semantics (DTS) presented in [3], Type Theory with Records (TTR) presented in [9], Unified Type Theory (UTT) presented in [16], and the framework we helped define, the Montagovian Generative Lexicon (MGL), presented in [32]. Several partial or complete implementations of those theories have been provided for demonstration purposes. Those tend to use logical or functional programming, or theorem provers such as Coq - [8] is an example.

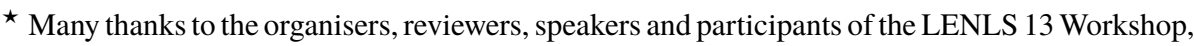
in the proceedings of which the first version of this publication appeared as [20]. Thanks also to the director and adjunct directors of the LaBRI for supporting this research.
} 
Concerning MGL, however, no real demonstration of the computational aspect has ever been provided. One of the stated goals of MGL was (paraphrasing slightly [32]) "to provide an integrated treatment from syntax to semantics extending existing analysers based on Montagovian semantics such as [25] with mechanisms for lexical semantics that are easily implemented in a typed functional programming language like Haskell'. Until the present publication, however, such implementations have been purely hypothetical, except for domain-specific analogues such as evoked in [26]. Our goal in this publication is to present an actual prototype implementation of the lexical semantics of that framework. For that purpose, we have used functional and object programming in Scala, but the paradigm and language of programmation are not critical elements. We also want to analyse what MGL can do using the (quite simple) computational mechanisms involved, as well as how those should be supplemented in order to provide useful treatments of semantics and pragmatics.

We detail some of the necessary data structures and algorithms used, what we learned from this implementation on the underlying logic properties of MGL, and sketch an architecture for simple knowledge representation that is necessary for the representation of certain lexical phenomena. The demonstrably functioning prototype illustrates both the validity of type-theoretic formulations of lexical meaning (including and not limited to MGL), and the deep interaction of lexical meaning with at least some sort of knowledge representation already evoked in [6].

\section{A MGL Prototype}

\subsection{The Montagovian Generative Lexicon}

MGL is a type-theoretic account of compositional lexical semantics that uses a calculus of semantic assembly called $\Lambda T Y_{n}$, an adaptation of the many-sorted logic $T Y_{n}$ (itself proposed in [28]) for the second-order $\lambda$-calculus, given in the syntax of Girard's System-F. MGL stays close to the usual Montague analysis by first performing a syntax-based analysis via proof-search, followed by the substitution of semantic main $\lambda$-terms to syntactic categories. Afterwards, lexical mechanisms are implemented in the meaning-assembly phase via a rich system of types based on ontologically different sorts and optional $\lambda$-terms that model lexical adaptation. The mechanisms, sketched in Fig. 1, are detailed in [24].

They can be roughly summarised as follows:

- First, the input utterance is super-tagged and analysed using categorial-grammar mechanisms. This is the only step of proof-search of the process, and yields a syntactic term whose components are syntactic categories. The lexicon is then used in standard Montagovian fashion to substitute semantic $\lambda$-terms to categories, yielding a main semantic term, typed with many sorts and the type $\mathbf{t}$ for propositions.

- Second, as a many-sorted logic is used, some type mismatches might occur. The direct correspondance between syntax and semantics is not guaranteed by the syntax as the arguments will be present in the correct number and position, but will not necessary be of the sort expected by the predicate. This allows the mechanisms of lexical semantics to engage and disambiguate between terms. 


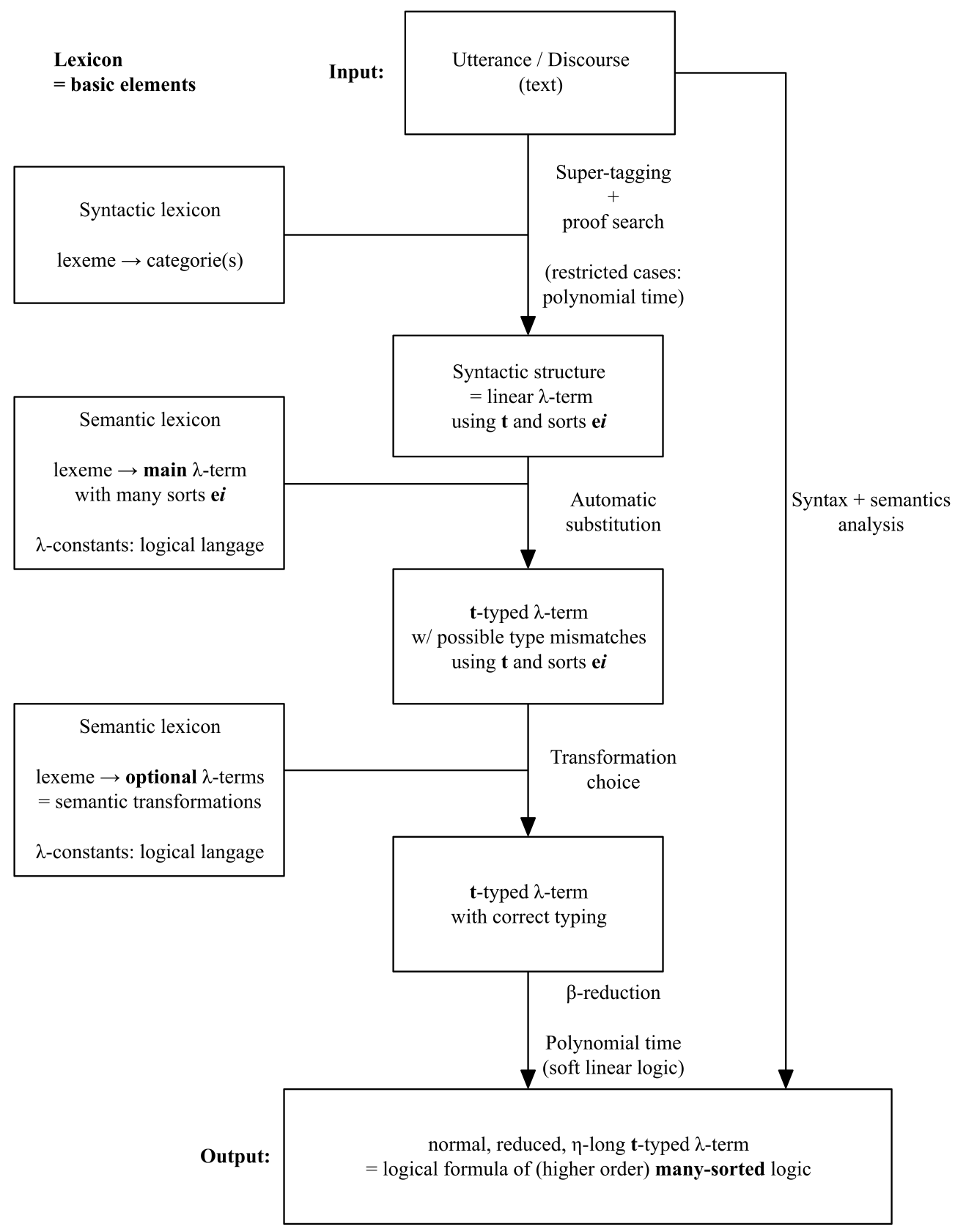

Fig. 1. MGL Process Summary 
To that effect, the lexicon provides optional $\lambda$-terms that are used as lexical transformations. These optional terms are inserted depending on their typing, and yield a $\lambda$-term with no type mismatches.

- Finally, $\beta$-reduction yields a normal, $\eta$-long $\lambda$-term of type $\mathbf{t}$ (the type of propositions), i. e. a logical formula that can be used in any usual semantic theories of meaning, including as model-theoretic and game-theoretic semantics.

As the first step, syntax-based analysis, is already well-studied and implemented (we use Type-Logical Grammars and Grail for this step, as given in [25]), the object of concern is the second step: given a term reflecting the syntactic structure of an utterance and a semantic lexicon, to construct a semantic $\lambda$-term in a many-sorted logic, making use of available transformations, and yielding a suitable formula. This is the object of our prototype implementation.

\subsection{Modelling Types and Terms}

The data structures and algorithms responsible for implementing the terms and types of $\Lambda T Y_{n}$ are the core mechanisms of the software. They are given as two Scala sealed abstract classes, TermW and TypeW, with a flat hierarchy of case classes implementing the various possible term and type categories; this simple categorisation allows us to easily construct and detect patterns of objects.

Terms and types are constructed as binary trees (abstractions and applications of more than one argument to a given term/type can be easily curryied):

- For terms:

- Leaves are AtomicTerms (constants), TermVarIds (variables) with an identifier and type, or specific constructs for MGL, Transformations and Slots.

- Inner nodes are TermBindings ( $\lambda$-abstracted terms), or TermApplications of a predicate term to an argument term.

- For types:

- Leaves are constant Sorts, pre-defined objects such as PropositionType for $\mathbf{t}$, or second-order variable identifiers TypeVarIds.

- Inner nodes are TypeFunctions between two types $A$ and $B$ (modelling $A \rightarrow$ $B$ ), or TypeApplications (modelling $A\{B\}$ ).

A simplified UML class diagram presents this straightforward architecture in Fig. 2.

Several algorithms are provided as specialised methods and class constructors in order to work with types and terms. They are mostly simple recursive tree-walking algorithms, making the most of memoisation when possible (e. g., lists of available ressources are incrementally built as terms and types are constructed in order to minimise computations). Algorithms include the type-checking of applications, comparison between types, automated $\alpha$-conversion of variables in order to prevent issues of scope, replacement of term and type variables, $\beta$-reduction, and the automated specialisation of types for polymorphic terms (i. e., a predicate with a type containing one or several type variables will be specialised to the correct types if applied to an argument with a compatible but specified type). In this prototype implementation, some degree of imperative programming is used in order to make some and optimisations easier. 


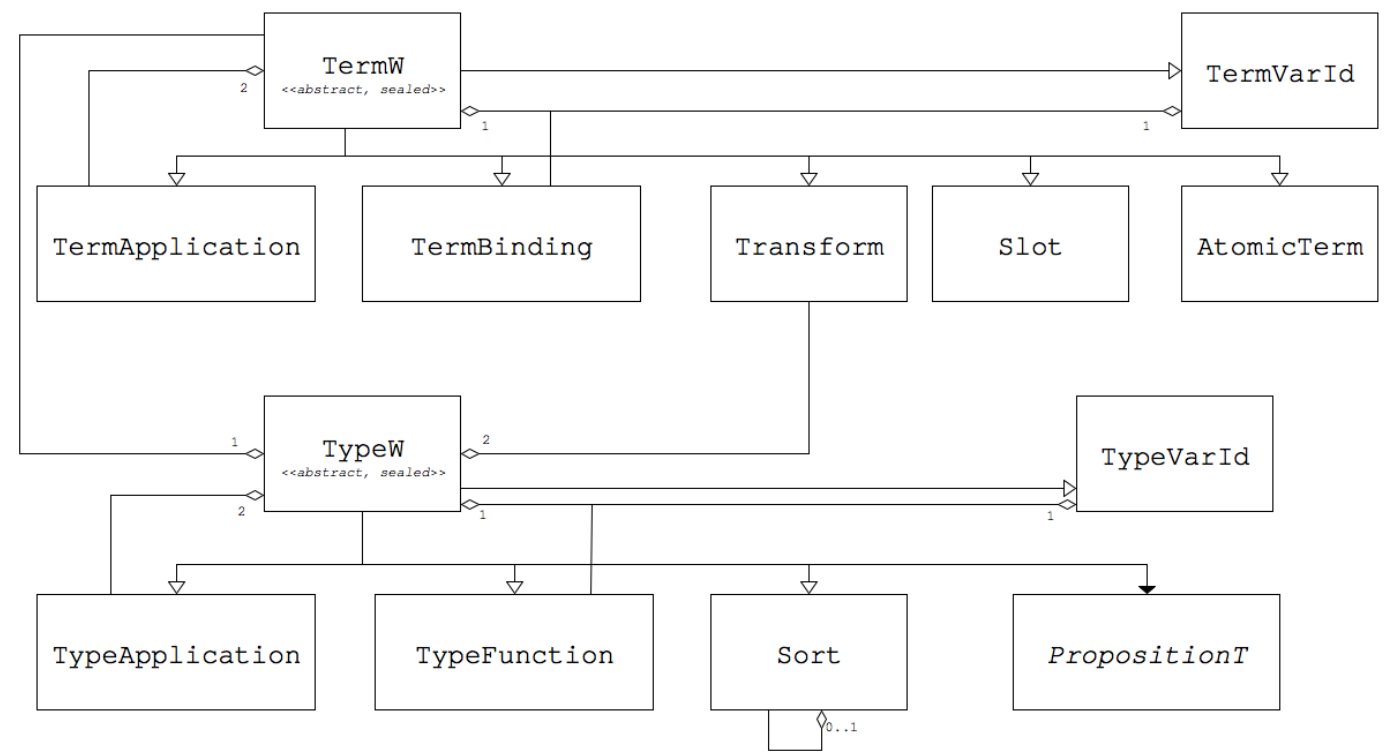

Fig. 2. Class diagram of the core package for terms and types.

It would be easy to convert the entire program to purely functional (or logical) programming. Most methods are linear in complexity (with exceptions in the adaptative mechanisms below); all algorithms are at most polynomial in time.

\subsection{Explicit Adaptation}

The core principle of MGL is to provide transformations as optional terms, on top of the main $\lambda$-term associated to each lexeme. A canonical example is the book is heavy and interesting. The usual, formal MGL analysis supposes three basic sorts:

- $R$ for readable materials,

- $\varphi$ for physical objects,

- I for informational contents;

the book can be modelled as the book ${ }^{R}$, heavy as heavy ${ }^{\varphi \rightarrow t}$, interesting as interesting $^{I \rightarrow \mathbf{t}}$. The example utterance is a case of co-predication, as two predicates are simultaneously asserted on two different facets (with different, incompatible sorts) of a same object, and MGL will resolve this by having the lexicon provide two optional terms associated with book in order to access these two facets: $f_{\text {phys }}^{R \rightarrow \varphi}$ and $f_{\text {info }}^{R \rightarrow I}$. 
In order to apply the co-predication, a specific operator, the polymorphic conjunction and, is required:

$$
\&^{\Pi}=\Lambda \alpha \Lambda \beta \lambda P^{\alpha \rightarrow \mathbf{t}} \lambda Q^{\beta \rightarrow \mathbf{t}} \Lambda \xi \lambda x^{\xi} \lambda f^{\xi \rightarrow \alpha} \lambda g^{\xi \rightarrow \beta} \text {. }\left(\text { and }^{\mathbf{t} \rightarrow \mathbf{t} \rightarrow \mathbf{t}}(P(f x))(Q(g x))\right)
$$

This yields, after suitable substitutions, application, and reduction, the term (and (heavy $\left(f_{\text {phys }}\right.$ book)) (interesting $\left(f_{\text {info }}\right.$ book $\left.)\right)$ ), which is normal and of type t.

In our implementation, there are several important differences with the formal account outlined above. As we distinguish between type constants and variables, there is no actual need to explicitly abstract (and $\Lambda$-bind) types. This is because the only second-order operation ever used in $\Lambda T Y_{n}$ is specialisation (i. e., the replacement or instantiation - of type variables). Moreover, second-order (type) variables are all introduced by $\lambda$-bound first-order (term) variables.

We also distinguish between term variables that are necessary for the definition of an abstracted term (such as $P, Q$ and $x$, the two predicates and the argument of the conjonction above) and adaptation slots, the positions where optional $\lambda$-terms (such as $f$ and $g$ above) can be inserted. This is because the optional terms can be provided by various different mechanisms, and might not be provided at all if the term is well-formed. There is no lexical adaptation taking place in utterances such as heavy and black rock; in that case, MGL provides an useful, if slightly redundant, $i d$ optional polymorphic term that can be inserted in order to get the identity on any type. Adding or removing adaptation slots is in fact a technical process analogue to type-raising or type-lowering in Montague semantics.

We provide optional terms (transformations) as Transforms, which are distinguished from other terms. Each term has a list of available transformations, constructed recursively from the leaves. The lexicon provides a list of transformations available to each lexeme, so that each atomic term has a collection of transformations. We also distinguish Slots for explicit adaptations; the list of slots is maintained during the construction of the terms. Our polymorphic and conjunction, also defined by the lexicon, then becomes:

$$
\begin{gathered}
\text { lambda } P^{\wedge}(B->t) \text {. lambda } Q^{\wedge}(G->t) . l \text { lambda } x^{\wedge} A .\left(\left(A n d^{\wedge}\{(t->(t->t))\}\right.\right. \\
\left.\left.\left(P^{\wedge}(B->t)\left(f^{\wedge}\{(A->B)\} x^{\wedge} A\right)\right)\right)\left(Q^{\wedge}(G->t) \quad\left(g^{\wedge}\{(A->G)\} x^{\wedge} A\right)\right)\right)
\end{gathered}
$$

(As above, $f$ and $g$ are adaptation slots, distinguished from other variables and not bound, while $P$ and $Q$ are $\lambda$-bound predicate variables.)

During the attempted resolution of the application of the conjunction to terms for heavy and interesting, the polymorphic and is specialised to sorts representing $\varphi$ and $I$, and cannot be reduced further with the application of the argument book. A further algorithm is provided in order to model the choice of transformations, trying to match all available transformations to the adaptation slots. As all permutations are considered, this is potentially the most costly computation taking place. The result is a list of possible interpretations (given as term applications with slots filled by transformations): there might be zero, one, or finitely many.

A further check on the list of terms obtained will filter those, if any, with a suitable typing, that will form the desired result(s). In the tests conduced with the input of 
the example, four interpretations where produced (one for every slot/transformation permutation), with only the correct one of a resolvable type (t):

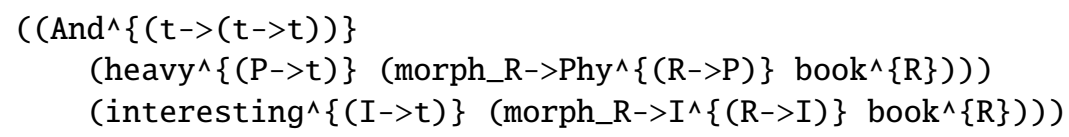

\subsection{Implicit Adaptation}

Polymorphic operators such as and, with explicit adaptation slots, are needed for co-predications. However, most lexical adaptations can take place implicitly, simply by reacting to a type mismatch and and applying any suitable transformation to resolve it. This requires to adapt the terms by adding correctly-typed and well-positioned adaptation slots. In the case of an application such as $\left(p^{A \rightarrow B} a^{C}\right)$, there are two possibilities to resolve the type mismatch: by adapting the predicate, yielding $\left.\left(\left(f^{(A \rightarrow B) \rightarrow(C \rightarrow B)}\right) p\right) a\right)$, or the argument, resulting in $\left(p\left(f^{C \rightarrow A} a\right)\right)$. In the slightly different case of a partial application $\left(\lambda x^{A} \cdot \tau a^{C}\right)$, in which the argument can be adapted as before (yielding $\left.\left(p\left(f^{C \rightarrow A} a\right)\right)\right)$, but the typing of the predicate might not be fully determined at the moment of the adaptation.

A procedure analyses such applications with type mismatches and no explicit adaptation slots, and inserts suitable, automatically generated adaptation slots, then proceeds as with explicit adaptations. For example, a simple term application such as $\left(P^{\wedge}\{(e->t)\} a^{\wedge}\{A\}\right)$, with a transformation $f_{-}\{A->e\}$ available to the atomic term a yields the straightforward (and only felicitous) interpretation (lambda $\left.x^{\wedge} A .\left(P^{\wedge}\{(e->t)\}\left(f_{-}\{A->e\}^{\wedge}\{(A->e)\} x^{\wedge} A\right)\right) a^{\wedge}\{A\}\right)$, that reduces to $\left(P^{\wedge}\{(e->t)\} \quad\left(f_{-}\{A->e\}^{\wedge}\{(A->e)\} a^{\wedge}\{A\}\right)\right.$.

Implicit adaptations are necessarily reduced to those simple cases. Trying to account automatically for co-predications would imply to try any possible permutation of types and transformations at all nodes of a term, which would be exponential in complexity; thus, the need for lexical operators with explicit adaptation slots such as the polymorphic and.

\subsection{Lexicalisation}

In addition to the core mechanisms, a tecto package provides support for a tectogrammatical/syntactic structure in the form of an unannotated binary tree of lexemes ; this serves as a factory for the input of already analysed text, and as a more streamlined form of output for adapted terms.

A lexicon package enables the storage of lexical entries that associate lexemes (as strings) to terms, complete with typing, transformations and ambiguities. Lexica can be merged, in order to have combine the treatment of different phenomena, treated as standalone modules, for complex sentences. Lexica also provide automated translations from a syntactic structure (a tecto term) to a semantic one (a TermW term, initially not adapted, reduced or even type-checked). Semantic terms can be presented either by a straightforward translation to syntactic terms, or printed to a string in the usual fully-parenthesed prefix notation with apparent typing (as in the examples of this article). 


\subsection{Phenomena Coverage}

Many lexical phenomena discussed in $[29,18]$ can be modelled using the simple mechanisms of $\Lambda T Y_{n}$ in their prototypal implementation given above; some others require additional mechanisms. The following is a short overview of how some classical phenomena are handled in MGL and our prototype.

Lexical adaptations, including alternations, meaning transfers, grinding, qualiaexploitation and "Dot-type"-exploitation are all supported by the adaptation mechanisms, as given previously.

Simple predications only require to have suitable transformations available, and to use the implicit adaptation mechanisms.

Co-predications require explicit adaptation using polymorphic operators (such as the higher-order conjunction "and" above). Theoretical grounds have been laid in $[18,32]$.

Constraints of application are required in order to perform co-predications correctly. As explained in [24], the simultaneous reference to different facets of a same entity can be infelicitous in some circumstances, such as the use of destructive transformations (grinding, packing) or metaphorical use of some words. Thus, the following co-predications are infelicitous to some degree:

- *The salmon was lighting-fast and delicious,

- ? Birmingham won the championship and was split in the Brexit vote.

In order to block such co-predications, we have proposed to place constraints on transformations in order to block their usage depending on the other transformations that have been used on the same term.

The first version of this system given in, e. g., [18], distinguishes between flexible (allowing all other facets) and rigid (blocking all other facets) transformations, as well as relaxable constraints depending on syntactic features. The latest version, given in [19], proposes a revised calculus named $\Lambda^{-0} T Y_{n}$, a system with terms of the linear intuitionistic logic as types, that (among other things) allow any arbitrary typedriven predicate to act as a constraint on the use of transformations, thus allowing the complex variability of felicity of co-predications with deverbals examined by [13] and detailed for MGL in [30].

In this prototype implementation, all transformations are equipped with a member function that can be defined as an arbitrary constraint, the default being the boolean constant true (that simply models flexible transformations). A compatibility oneon-one check of all transformations can be performed using every constraint. As the constraint can effectively be any function, the precision is the same as in [19].

Ontological inclusion, called type accommodation in [29] and modelling the lexical relation of hyponymy (as evoked with different solutions in other sub-typing accounts, such as [4]), can be supported by tweaking the system of sorts. The theoretical and empirical basis for doing so are discussed in [23], in which we argue that coercive sub-typing is an accurate and helpful mechanism for resolving ontological inclusion, but no other lexical phenomena; the latter are implemented using word-level transformations. In order to support sub-typing, each sort can be defined with an optional parent sort. A careful review of the typing comparison mechanism will then be enough in order to support sub-typing. 
This is not implemented yet (it requires a refactoring of the notion of equality for sorts), but does not require (much) additional processing power.

Performative lexical adaptations, such as quantification, Hilbert operators for determiners, and the alternate readings of plurals and mass nouns, are supported as far as the meaning assembly phase is concerned. However, in order to be useful, this category of lexical phenomena (as well as hypostasis and several others) require additional mechanisms in order to incorporate the knowledge gathered from the analysis of the sentence into the logical representation. The basic architecture is supported, but mechanisms of resolution remain preliminary and will be discussed next, especially in Section 3.3.

\section{Layers of Lexica and Knowledge Representation}

\subsection{The Additional Layers}

Theories of semantics deriving from [29] generally encompass some degree of common sense world knowledge. For example, it is considered known that a committee (and other such group nouns) is made of several people, and is thus a felicitous argument of predicates requiring a plural argument such as to meet. It is also known that engines are part of cars, and that predicates such as powerful or fuel-guzzling can apply to cars via their constitutive quale; all such "common-sense metaphysics" have been part of generative lexical theory from the start, as detailed by [2]. It has been argued (e. g. in [11]) that such complex knowledge does not belong in a semantic lexicon; we will ignore such claims, paraphrasing Im and Lee from [12] in defining semantics to be the meaning conveyed by an utterance to a competent speaker of the language in itself, excluding, for instance, the specific situation in which the utterance is made, but including any previous discourse. In this view the full contents of, for example, a given fairy tales, should be able to be described within semantics, while texts such as political essays will probably require additional knowledge about the position of the author and the specifics of the period of writing. From our point of view, the lexicon includes that minimal knowledge as part of the semantic terms and types involved. To design a complete tool for type-theoretic lexical semantics, we must first complete the careful definition of various lexica that can convey the necessary, elementary world-knowledge for each word. A lexicon for general use will associate to all relevant lexemes their semantics (in the form of main and optional $\lambda$-terms) as can be given in a dictionary of a language. However, there are two arguments to be made for additional lexica beyond the general language lexicon.

First are the specific lexica, that detail vocabularies relevant only to a community, such as professional jargons, local dialects, and other linguistic constructs specific to small groups of people; and/or words or word uses restrained to a specific literary universe, such as fairy tales, space opera, mythology, politic speeches, etc. Such lexica are activated on an as-needed basis, and are more specific than the general-use lexicon.

Lexical semantics also requires a lexicon used for the current enunciation. A competent speaker of any language is able to use generative mechanisms in order to introduce new lexical concepts, either by the use of a new word, the meaning of which can be inferred from context and morphology, or by creative use of an existing word. 
In our view, the lexicon of the enunciation starts empty and can be augmented when the analysis of the discourse encounters words that are not present in the current active lexica. We think that such mechanisms can enable the learning of lexical semantic data. In addition to these lexical layers of meaning, we tend to implement different lexical phenomena using different lexica for simplicity's sake, and create a merged lexicon from every relevant one when processing text.

\subsection{Individuals, Facts and Contexts}

To summarise our argument in Section 3.1 above, in addition to mostly static lexical data, some sort of knowledge representation is needed to process even simple lexical phenomena such as collective and distributive readings for plurals. Namely, we need to keep track of the individuals mentioned in a given discourse, and of the facts asserted of those individuals. To be complete, we would also need to keep track of agents, in order to model dialogues or multiple points of view in which certain agents assert certain facts. Our implementation prototype supports individuals, as atomic terms of type $A$ (for named entities: human agents, towns...), as well as some individuals as atomic terms of type $A \rightarrow \mathbf{t}$ (for common nouns, that can be resolved to a specific individual of type $A$ by the means of an Hilbert-based determiner) for any suitable sort $A$. This dual typing for individual is adopted by MGL for complete compatibility with the classical Montagovian analysis, and is well-suited for the treatment of many phenomena, using operators inspired by Hilbert for the representation of determiners (as given in [22]) in order to select a specific individual from a common noun. We think that this approach is justified; however, this is not the chosen modelisation for other type-theoretic accounts of lexical semantics, and the implementation provided is easily adapted to other types for common nouns and individuals. See [24] for a detailed discussion of our choice of types and [17] for a contrasting opinion.

We also account for facts, as predicates (TermBindings or atomic terms) of type $\alpha \rightarrow \mathbf{t}$ for any arbitrarily complex type $\alpha$, that are used in a term application, and apply to an individual. In the analysis of a term, individuals and types are extracted and added to the context of enunciation. The hierarchy of lexical layers given above can be implemented as a hierarchy of contexts, some containing initial individuals and facts relevant to each lexicon; in a such complete system, the context of the real world would, to resolve the paradox mentioned in [33], include the fact that there is no King of France (and therefore that The king of France is bald, while grammatical, is not felicitous because there are no qualifying referents for the entities described, and thus cannot be assigned a truth value). Such contexts are specific objects (aggregating individuals, facts and a related lexicon) in our implementation.

\subsection{The Parsing-Knowledge Loop}

We use a specific lexicon to list some common semantic terms for quantifiers (universal/existential, needed for some classical interpretations), counting terms (needed for plurals), logical constants (for truth-valued semantic interpretations of grammatical constraints) and Hilbert operators (used for the smooth modelling of determiners, as detailed in e. g. [31] and more recently in [22]). 
Other lexica can make use of these terms in order to construct, for instance, Linkbased semantics for plurals (originally given in [15]), using lexical transformations as detailed in [21]. Some functions associated to the logical lexicon then resolve the glue operators, given a term and a context. This updated process of analysis is given in Fig. 3 .

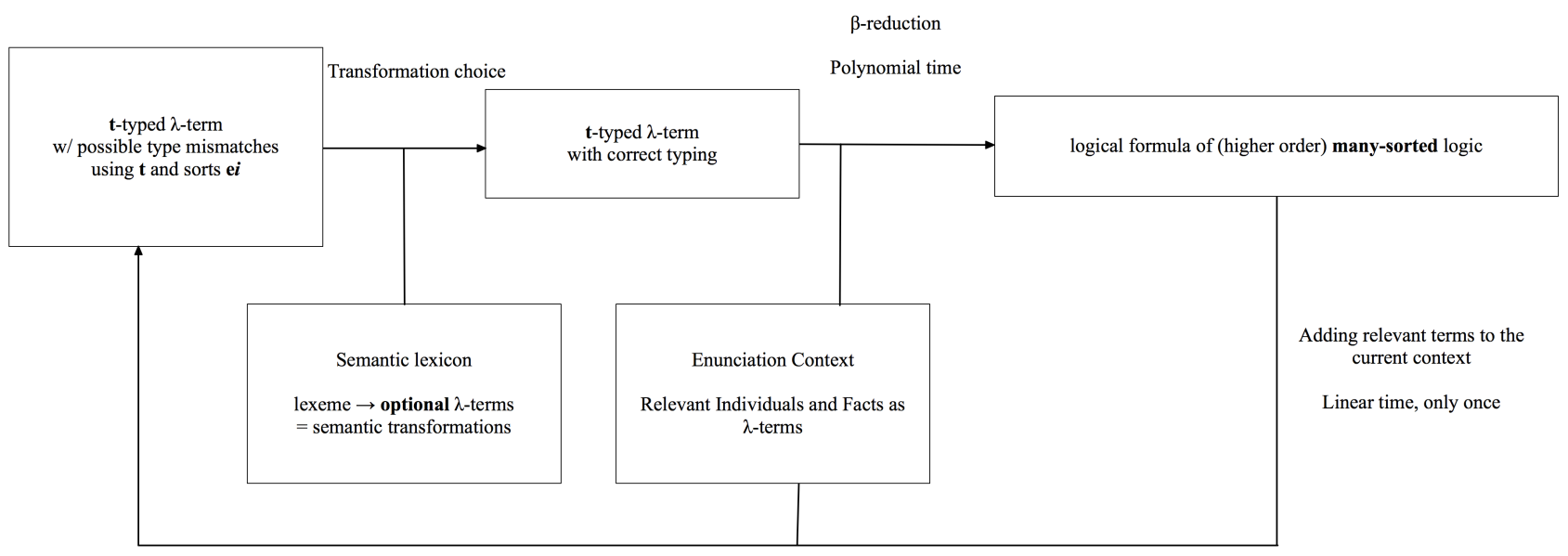

Fig. 3. Parsing-Knowledge Representation Feedback

To explain what the analysis of plural readings in MGL entail, consider the following example from [21] :

- Jimi and Dusty met is analysed as

$\left|\lambda y^{e} .(y=j) \vee(y=d)\right|>1 \wedge \operatorname{meet}\left(\lambda y^{e} .(y=j) \vee(y=d)\right)$.

One elementary issue is that the predicate met applies to group individuals (such as a committee) and constructions made of more than one individuals (such as Jimi and Dusty) but not to singular individuals (such as a student). Thus, the lexical entry for the predicate is $\lambda P^{e \rightarrow t} .|P|>1 \wedge$ meet $(P)-$ a logical conjunction with a cardinality operator.

Those two simple elements can be defined in System-F (the calculus in which $\Lambda T Y_{n}$, the logic of MGL, is implemented). The issue is that, in order for our system to infer correctly that Jimi and Dusty are two different individuals, and thus that the above term resolves to meet $\left(\lambda y^{e} .(y=j) \vee(y=d)\right)$, we must use processing power beyond the simple construction and reduction of terms: a minimal system of knowledge representation and logical inference.

Within our architecture encompassing individuals and facts, and with a functional lexicon for logical connectives (including the logical and operator of that example), as well as quantification and counting (including the cardinality operator), this example can be treated.

However, this requires a given term to be parsed at least twice. The first time, the syntactic structure is converted into a semantic term and lexical transformations are applied. 
The second time, facts that emerge from the transformations, such as alternate distributive/collective readings for plural predicates, are added to the lexicon, and the logical lexicon can be used in order to process the operators that have been introduced. Our prototype implementation does not incorporate such feedback yet, as the first step can result in several different interpretations; this remains a work in progress. As a result, straightforward composition for plurals are tentatively supported (such as in the previous example), but ambiguous covering readings for plurals are not yet available.

\subsection{World Sense Acquisition}

An enunciation-context lexicon that is filled with individuals and facts inferred from the primary semantic analysis can serve, in a limited way, to account for words that are absent from the lexicon. Such words, syntactically placed in the position occupied by individuals or facts, will be added as primary entities to the lexicon, and their precise typing inferred from the predicates they are applied to. The typing can then be refined as the new lexeme is used again. An elementary mechanism should be enough to have a correct (if completely underspecified) representation from Lewis Carroll's Jabberwocky.

Of course, most competent human speakers also use morphosyntactic inference to attach at least some degree of connotative meaning to the words being proposed (e. g., Star Wars's plasteel can be inferred as a fictive material somehow combining the characteristics of plastics and steel by any English speaker). This is completely beyond the power of our early software, and simple syntax-and-typing inferences.

A first easy step is to have the process of meaning assembly outline which lexemes are not in the lexicon, and use human input for correcting the precise types and terms associated. Promising automated strategies for learning more about new lexemes, or lexemes used in a creative way, are also explored in richly typed settings by [7].

\subsection{Quantificational Puzzles}

The process of counting, quantifying and selecting entities using Hilbert operators can also shed some light on the quantificational puzzles mentioned in [1] and several other related works. The issue with having universal quantification used together with co-predication on multi-faceted entities can be seen in examples such as :

- There are five copies of War and Peace and a copy of an anthology of Tolstoi's complete works on the shelf.

What is the answer to questions such as How many books...?

What exactly is the type of book in such questions?

- I read, then burnt, every book in the attic.

The entities being predicated form two different sets.

In order to resolve such quantificational puzzles satisfactorily, the methods for counting and quantifying must be adapted to each predicate, and only apply to individuals of the appropriate type. For our purpose, this implies a close monitoring of the entities introduced by lexical transformations and their context of appearance.

As the quantifiers are adapted to the transformed and original entities, maintaining expected truth values for straightforward sentences poses a challenge. This is also a work in progress. 


\section{Results}

\subsection{A Fragment of Second-Order}

With this prototype software, we have proven that MGL can actually be computationally implemented. This was not really in doubt, but the way that the combination of types and terms are implemented illustrates that the time and space complexity of most of the process is limited: the algorithms used are mostly linear tree walks, with a few quadratic worst-case operations.

The most complex step is the choice of optional terms for adaptation slots, of complexity $|t| \times|s| \times n$ at worst (the product of the number of optional terms available, adaptation slots, and length of the term); the hypothesis behind MGL is that the number of available optional terms at any point remains "manageable". Thus, the step not actually implemented in this prototype (but for which many implementations exist), syntactic analysis, is the costliest of the steps detailed in Fig. 1, and the complete process of parsing is polynomial in time, as explained in [24].

MGL accounts such as [32] point out that the whole expressive power of second-order $\lambda$-calculus is not used, and that every possible operation could be implemented using first-order terms if all possible adaptations were listed at each step (which is syntactically much longer to write). Indeed, our implementation only supports the single second-order operation of type specialisation (by distinguishing type variables from other types and using pattern matching to recognise and rewrite types), which is required for having polymorphic terms. There are no features of $\Lambda T Y_{n}$ that require additional power: subtyping can be implemented by an optional parent field in Sorts, arbitrary complex on co-predications are supported by including a check on transformations that can be any arbitrary function, quantification, counting and Hilbert operators can be included. . .

Formally, it has been pointed out by $\mathrm{Ph}$. de Groote (pc.) that the smallest type calculus encompassing the features provided by $\Lambda T Y_{n}$ is a simply-typed calculus supporting type collections and sub-typing, such as the typing system used by OCaML (http://ocaml.org/). Moreover, the many efforts made by proponents of other related type-theoretic accounts of generative lexical semantics to provide implementations of their own theories reinforce our belief in the computational feasibility of such analyses. The chain of treatment sketched in Fig. 1 and detailed in [24], and the computational steps provided in the present paper, are very similar to, for instance, the process detailed in [14] for Dependent Type Semantics. The many differences between the theories and representations notwithstanding, the operational steps used to analyse similar phenomena are nearly identical (despite the research process on both accounts being parallel and candid, and the presentation of the results having occurred on the same day). Such spontaneous convergence between theories is, in our view, an indication of the pertinence of both accounts.

\subsection{Minimal Processing Architecture}

Our prototype implementation includes the skeleton of an architecture that represents the individuals, facts and agents appearing during the semantic analysis. 
This goes beyond the straightforward process of producing a logical representation for an utterance, as some of the terms of that logical representation might be analysed differently depending on the context; we argue that this process should still be part of a semantic analysis. The individuals, facts and agents are stored in objects called contexts, organised in a hierarchy that includes the most specific context (modelling the analysis of the current discourse), universe-specific contexts (describing whether the discourse is part of a fictional, historical or activity-specific setting), dialect- and language-specific contexts, each associated to an appropriate lexicon.

A complete analysis would minimally involve the construction of the logical representation of an utterance, the update of the enunciation context with individuals and facts introduced by that utterance, and a re-interpretation of the logical representation in the active contexts. This minimal processing architecture can be completed with no difficulties; our implementation includes relevant data structures and algorithms, but requires significant work on examples of performative lexica in order to be thoroughly tested.

\subsection{Perspectives}

This prototype implementation has already served its primary purpose: to illustrate that MGL can be computationally implemented, and that the examples usually given with the theory actually work. As it stands, however, this implementation is more of a proof of concept than useful software.

To be actively used by the community, more work would be required to give it an helpful interface, both for the user and for existing analysers; we also would like to convert from and to representations of the other most active type-theoretic accounts of lexical semantics. The knowledge-representation architecture remains a work in progress, and requires solid efforts in order to correspond to our ambitions. The completion of this software is not, however, an end in itself.

In fact, what MGL (and other related accounts of lexical semantics) really requires in order to be useful is a large-cover library of types and terms. The analyses, whether formal or computational, are justified by toy linguistic examples or on domain-restricted phenomena; without a significant step in the definition of a system of sorts and types, and subsequently of a large, semantically rich lexicon, type-theoretic proposals have very little value compared to, e. g., "deep" neural techniques trained using massive corpora. The prototype software presented in this publication is a nice illustration of the possibilities provided by MGL, but our main hope is that it can help to build software that can learn types and lexemes.

The first step will not be on the software side but, as suggested in [24], the establishment of a linguistically motivated kernel system of sorts.

\section{References}

1. Asher, N.: Lexical Meaning in Context: A Web of Words. Cambridge University Press (Mar 2011)

2. Asher, N., Pustejovsky, J.: Word Meaning and Commonsense Metaphysics. Semantics Archive (Aug 2005) 
3. Bekki, D.: Dependent Type Semantics: An Introduction. In: Christoff, Z., Galeazzi, P., Gierasimczuk, N., Marcoci, A., Smet, S. (eds.) Logic and Interactive RAtionality (LIRa) Yearbook 2012. vol. I, pp. 277-300. University of Amsterdam (2014)

4. Bekki, D., Asher, N.: Logical polysemy and subtyping. In: Motomura, Y., Butler, A., Bekki, D. (eds.) JSAI-isAI Workshops. Lecture Notes in Computer Science, vol. 7856, pp. 17-24. Springer (2012)

5. Bierwisch, M.: Wördliche Bedeutung - eine pragmatische Gretchenfrag. In: Grewendorf, G. (ed.) Sprechakttheorie und Semantik, pp. 119-148. Surkamp, Frankfurt (1979)

6. Bosch, P.: The bermuda triangle: Natural language semantics between linguistics, knowledge representation, and knowledge processing. In: Text Understanding in LILOG, Integrating Computational Linguistics and Artificial Intelligence, Final Report on the IBM Germany LILOG-Project. pp. 243-258. Springer-Verlag, London, UK, UK (1991), http://dl . acm. org/citation. cfm?id=647415.725191

7. Breitholtz, E.: Are Widows Always Wicked? Learning concepts through enthymematic reasoning. In: Cooper, R., Retoré, C. (eds.) ESSLLI proceedings of the TYTLES workshop on TYpe Theory and LExical Semantics. Barcelona (Aug 2015)

8. Chatzikyriakidis, S., Luo, Z.: Natural language inference in coq. J. of Logic, Lang. and Inf. 23(4), 441-480 (Dec 2014), http: //dx . doi .org/10 . 1007/s10849-014-9208-x

9. Cooper, R.: Copredication, dynamic generalized quantification and lexical innovation by coercion. In: Fourth International Workshop on Generative Approaches to the Lexicon (2007)

10. Cruse, D.A.: Lexical Semantics. Cambridge, New York (1986)

11. Fodor, J.A., Lepore, E.: The emptiness of the lexicon : Reflections on James Pustejovsky's The Generative Lexicon. Liguistic Inquiry 29(2) (1998)

12. Im, S., Lee, C.: A developed analysis of type coercion based on type theory and conventionality. In: Cooper, R., Retoré, C. (eds.) ESSLLI proceedings of the TYTLES workshop on TYpe Theory and LExical Semantics. Barcelona (Aug 2015)

13. Jacquey, E.: Ambiguïtés lexicales et traitement automatique des langues : modélisation de la polysémie logique et application aux déverbaux d'action ambigus en français. $\mathrm{Ph} . \mathrm{D}$. thesis, Université de Nancy 2 (2001)

14. Kinoshita, E., Mineshima, K., Bekki, D.: An analysis of selectional restrictions with Dependent Type Semantics. In: Proceedings of the 13th International Workshop on Logic and Engineering of Natural Language Semantics (LENLS13). pp. 100-113 (Nov 2016)

15. Link, G.: The logical analysis of plurals and mass terms: A lattice-theoretic approach. In: Portner, P., Partee, B.H. (eds.) Formal Semantics - the Essential Readings, pp. 127-147. Blackwell (1983)

16. Luo, Z.: Contextual analysis of word meanings in type-theoretical semantics. In: Pogodalla, Prost (eds.) LACL'11 Proceedings of the 6th international conference on Logical aspects of computational linguistics, pp. 159-174. Springer-Verlag Berlin, Heidelberg (2011)

17. Luo, Z.: Common nouns as types. In: Béchet, D., Dikovsky, A. (eds.) Logical Aspects of Computational Linguistics, Lecture Notes in Computer Science, vol. 7351, pp. 173-185. Springer Berlin Heidelberg (2012)

18. Mery, B.: Modélisation de la Sémantique Lexicale dans le cadre de la Théorie des Types. Ph.D. thesis, Université de Bordeaux (Jul 2011)

19. Mery, B.: Lexical Semantics with Linear Types. In: NLCS '15, the Third Workshop on Natural Language and Computer Science. Kyoto, Japan (Jul 2015)

20. Mery, B.: Lessons from a Prototype Implementation of Montagovian Lexical Semantics. In: Proceedings of the 13th International Workshop on Logic and Engineering of Natural Language Semantics (LENLS13). pp. 73-85 (Nov 2016)

21. Mery, B., Moot, R., Retoré, C.: Computing the Semantics of Plurals and Massive Entities using Many-Sorted Types. In: Murata, T., Mineshima, K., Bekki, D. (eds.) New Frontiers in 
Artificial Intelligence JSAI-isAI 2014 Workshops, LENLS, JURISIN, and GABA, Kanagawa, Japan, October 27-28, 2014, Revised Selected Papers, Lecture Notes in Artificial Intelligence, vol. 9067, p. 357. Springer-Verlag Berlin Heidelberg (2015), https://hal.inria.fr/ hal-01214435

22. Mery, B., Moot, R., Retoré, C.: Typed Hilbert Operators for the Lexical Semantics of Singular and Plural Determiner Phrases. In: Epsilon 2015 - Hilbert's Epsilon and Tau in Logic, Informatics and Linguistics. Montpellier, France (Jun 2015)

23. Mery, B., Retoré, C.: Are books events ? Ontological Inclusions as Coercive Sub-Typing, Lexical Transfers as Entailment. In: LENLS '12, in jSAI 2015. Kanagawa, Japan (Nov 2015)

24. Mery, B., Retoré, C.: Classifiers, Sorts, and Base Types in the Montagovian Generative Lexicon and Related Type Theoretical Frameworks for Lexical Compositional Semantics. In: Chatzikyriakidis, S., Luo, Z. (eds.) Modern Perspectives in Type-Theoretical Semantics, Studies in Linguistics and Philosophy, vol. 98, pp. 163-188. Springer International Publishing (2017)

25. Moot, R.: Wide-coverage French syntax and semantics using Grail. In: Proceedings of Traitement Automatique des Langues Naturelles (TALN). Montreal (2010)

26. Moot, R., Prévot, L., Retoré, C.: A discursive analysis of itineraries in an historical and regional corpus of travels. In: Constraints in discourse. $p$. http://passage.inria.fr/cid2011/doku.php. Ayay-roches-rouges, France (Sep 2011), http: //hal . archives-ouvertes. fr/hal-00607691/en/

27. Moravcsik, J.M.: How do words get their meanings ? The Journal of Philosophy LXXVIII(1) (Jan 1982)

28. Muskens, R.: Meaning and Partiality. In: Cooper, R., de Rijke, M. (eds.) Studies in Logic, Langage and Information. CSLI (1996)

29. Pustejovsky, J.: The Generative Lexicon. MIT Press (1995)

30. Real-Coelho, L.M., Retoré, C.: A generative montagovian lexicon for polysemous deverbal nouns. In: 4th World Congress and School on Universal Logic - Workshop on Logic and Linguistics. Rio de Janeiro (2013)

31. Retoré, C.: Sémantique des déterminants dans un cadre richement typé. CoRR abs/1302.1422 (2013), http://arxiv.org/abs/1302 . 1422

32. Retoré, C.: The Montagovian Generative Lexicon Lambda TYn: a Type Theoretical Framework for Natural Language Semantics. In: Matthes, R., Schubert, A. (eds.) 19th International Conference on Types for Proofs and Programs (TYPES 2013). Leibniz International Proceedings in Informatics (LIPIcs), vol. 26, pp. 202-229. Schloss Dagstuhl-Leibniz-Zentrum fuer Informatik, Dagstuhl, Germany (2014), http://drops . dagstuhl . de/opus/volltexte/ $2014 / 4633$

33. Russell, B.: On denoting. Mind 14(56), 479-493 (1905), http://www . jstor . org/stable/ 2248381 